\title{
La dinámica del miedo: la cascada defensiva
}

\author{
Jaime Vila, Pedro Guerra, Miguel A. Muñoz, Pandelis Perakakis, \\ Luis Carlos Delgado, Marlen Figueroa, Sofía Mohamed
}

\author{
Universidad de Granada
}

Disponible online 22 diciembre de 2009

\begin{abstract}
El estudio de las reacciones defensivas constituye una de las principales vías de conocimiento de los mecanismos psicológicos y neurofisiológicos del miedo y la ansiedad. Los antecedentes de estas investigaciones se encuentran en los trabajos de Pavlov sobre los reflejos incondicionados de orientación y defensa y en los trabajos de Cannon sobre las respuestas defensivas de lucha o huida, dando lugar a dos aproximaciones diferentes: la aproximación atencional y la aproximación motivacional. Estas dos tradiciones han sido difíciles de reconciliar en el pasado. Sin embargo, en los últimos años se han ido acumulando nuevos datos sobre las respuestas defensivas que están facilitando la integración de ambas tradiciones. Una parte de estos datos se refiere a la respuesta cardíaca. Las investigaciones recientes demuestran que la defensa cardíaca es un patrón complejo de cambios cardíacos con dos componentes acelerativo/ desacelerativos secuenciales, con mediación fisiológica tanto simpática como parasimpática, y con significación psicológica tanto atencional como motivacional. En este contexto, se ha propuesto un modelo atencional-motivacional de la defensa cardíaca que postula una fase atencional defensiva -análoga a la respuesta de inmovilidad- asociada a la primera aceleración/desaceleración y una fase motivacional defensiva -análoga a la respuesta de lucha/huida- asociada a la segunda aceleración/desaceleración.
\end{abstract}

Palabras clave: Cascada defensiva, miedo, defensa activa, defensa pasiva, frecuencia cardiaca, atención

The study of defense reactions has become in the last years one of the most relevant ways to advance knowledge on the psychological and neurophysiological mechanisms of fear and anxiety. The antecedents of these studies are rooted in the work of Pavlov on the unconditioned reflexes of orienting and defense and on the work of Cannon on the fight or flight response, giving rise to two different approaches to defense: the attentional and the motivational. These two approaches have been difficult to reconcile in the past. However, in the last years new data have been accumulated on defense reactions which are facilitating the integration of both traditions. Part of these data refers to the cardiac defense response. Recent research in this area has shown that cardiac defense is a complex pattern of heart rate changes with two accelerative/decelerative sequential components, with both sympathetic and parasympathetic physiological mediation, and with both attentional and motivational significance. In this context, an attentional-motivational model of cardiac defense has been proposed. The model postulates an attentional defense phase -analogous to the freezing response- associated with the first acceleration/deceleration and a motivational defense phase -analogous to the fight/ flight response- associated with the second acceleration/deceleration.

Keywords: Defense cascade, fear, active defense, pasive defense, heart rate, attention

Las investigaciones descritas en el presente trabajo han sido financiadas por la Junta de Andalucía (Grupo HUM-388) y el Ministerio de Educación y Ciencia (proyectos SEJ2004-07956/PSI y PSI2008-04372).

Correspondencia: Jaime Vila. Facultad de Psicología. Departamento de Personalidad, Evaluación y Tratamientos Psicológicos. Universidad de Granada, Campus de la Cartuja, 18071, Granada. E-mail: jvila@platon.ugr.es 
El concepto de defensa y su relevancia en el estudio del miedo y la ansiedad

El concepto de defensa hace referencia a la reacción fisiológica de los organismos ante la presencia de peligro o amenaza. Reacciones defensivas típicas incluyen la inmovilidad, el sobresalto, el desmayo y la respuesta de lucha/huida. La función protectora de estas reacciones es evidente. Sin embargo, si son demasiado intensas o prolongadas, las respuestas defensivas pueden convertirse en un serio riesgo para la salud tanto física como psicológica (Dienstbier, 1989; Lovallo y Gering, 2003; Turner, 1994).

El miedo y la ansiedad son las respuestas emocionales ante la presencia de peligro o amenaza estando, por tanto, estrechamente relacionadas con el concepto de defensa. Los avances en el estudio neurocientífico del miedo y la ansiedad proceden de investigaciones con animales utilizando reacciones defensivas (Blanchard \& Blanchard, 1989; Davis, 1992; Fanselow, 1994; LeDoux, 2000). Los paradigmas más utilizados en el estudio de los circuitos cerebrales del miedo (condicionamiento, sensibilización y potenciación del miedo) siempre incluyen estimulación inductora de reacciones defensivas con el fin de investigar las vías cerebrales de la respuesta de miedo. La investigación en humanos también ha utilizado con frecuencia estimulación intensa o aversiva para estudiar la modulación emocional de reflejos protectores como el sobresalto o la inmovilidad postural (Azevedo et al., 2005; Lang, Davis y Öhman, 2000; RuizPadial y Vila, 2007).

Los trastornos de la ansiedad también están estrechamente relacionados con el concepto de defensa. Tal vez la evidencia más dramática de la relevancia de las reacciones defensivas en el desarrollo de ansiedad patológica procede de estudios clínicos sobre el trastorno de estrés postraumático como consecuencia de violencia extrema (Schauer, Neuner y Elbert, 2005). Sin embargo, en estos casos, como en cualquier situación donde surge un peligro real, es difícil hablar de la reacción defensiva como una entidad única. Se produce, más bien, una secuencia dinámica o cascada de reacciones defensivas que varían en función del tipo y severidad del peligro, de su proximidad espacial y temporal, y del éxito o fracaso de las respuestas defensivas iniciales (Fanselow, 1994; Lang, Bradley y Cuthbert, 1997; McNoughton y Corr, 2004).

Aproximaciones tradicionales al estudio de la defensa cardíaca

Los antecedentes históricos del concepto de defensa se encuentran en los trabajos de Pavlov (1927) sobre los reflejos incondicionados de orientación y defensa y en los trabajos de Cannon (1929) sobre las respuestas defensivas de lucha o huida. Pavlov utilizó el término reflejo defensivo para referirse a respuestas fisiológicas protectoras ante estímulos nocivos como la retirada de la mano ante un calambre, el parpadeo ante un soplo de aire o el vómito ante comida en mal estado. Can- non, por su parte, utilizó el término defensa para referirse a la respuesta de lucha o huida, una respuesta cardiovascular mediada simpáticamente, dirigida a facilitar conductas adaptativas ante situaciones de peligro.

Con Pavlov y Cannon se inician las dos grandes tradiciones en la investigación psicológica de las reacciones defensivas: la tradición cognitiva y la tradición motivacional. La tradición cognitiva (Graham, 1992; Lacey y Lacey, 1974; Sokolov, 1963) asume que los cambios cardíacos en respuesta a estímulos ambientales reflejan mecanismos atencionales y perceptivos dirigidos a facilitar o inhibir el procesamiento de los estímulos. El reflejo de orientación (una desaceleración de la frecuencia cardíaca ante estímulos novedosos) facilita la atención y percepción del estímulo, mientras que el reflejo de defensa (una aceleración de la frecuencia cardíaca ante estímulos intensos o aversivos) reduce la atención y la percepción como una forma de protección ante el estímulo aversivo. Por su parte, la tradición motivacional (Obrist, 1981; Selye, 1956; Steptoe y Vögele, 1991) asume que los cambios cardíacos en respuesta a las demandas ambientales reflejan mecanismos metabólicos dirigidos a proporcionar al cuerpo la energía necesaria para llevar a cabo comportamientos adaptativos. Si la conducta apropiada es permanecer pasivo y quieto, entonces la respuesta cardíaca es una desaceleración de la frecuencia cardíaca. Si la conducta apropiada es reaccionar activamente, tanto psicológica como físicamente, entonces la respuesta cardíaca es una aceleración de la frecuencia cardíaca. Esta distinción entre defensa activa/pasiva es similar a la distinción propuesta por varios investigadores entre estilos defensivos proactivos/reactivos, los primeros caracterizados por la tendencia a reaccionar defensivamente con conductas de evitación activa y agresión, y los segundos por reaccionar defensivamente con conductas de evitación pasiva e inmovilidad (Frankenhaeuser, 1986; Koolhaas et al., 1999).

Estas dos tradiciones han sido difíciles de reconciliar en el pasado. Desde la perspectiva cognitiva, la significación funcional de la defensa cardíaca se entiende como un mecanismo atencional opuesto al mecanismo atencional propio del reflejo de orientación. Desde la perspectiva motivacional, la significación funcional de la defensa cardíaca se entiende como un mecanismo de movilización energética del organismo opuesto al estado de relajación. Sin embargo, en los últimos años se han ido acumulando nuevos datos sobre las respuestas defensivas que no sólo están facilitando la integración de ambas tradiciones dentro de un nuevo modelo -el modelo de la cascada defensiva- sino que, además, están contribuyendo a entender mejor los mecanismos psicológicos y neurofisiológicos implicados en las reacciones defensivas. Una parte de estos datos se refiere a la respuesta cardíaca de defensa. Las investigaciones recientes en este campo demuestran que la respuesta cardíaca de defensa, en contra de los supuestos tradicionales (aceleración cardíaca, mediada simpáticamente y con significación cognitiva o motivacional), es un patrón complejo de cambios cardíacos, 
con componentes acelerativos y desacelerativos secuenciales, con mediación fisiológica tanto simpática como parasimpática, y con significación psicológica tanto cognitiva como motivacional (Vila et al., 2007). A conclusiones similares se ha llegado en el contexto de otras respuestas defensivas: el reflejo de sobresalto (Lang, Bradley y Cuthbert, 1997) y la respuesta de inmovilidad (Azevedo et al. 2005). Ambas respuestas pueden estar simultáneamente moduladas por factores atencionales y motivacionales.

\section{La cascada defensiva}

El modelo de la cascada defensiva entiende que las reacciones defensivas siguen un proceso secuencial con fases iniciales en las que predominan los factores atencionales dirigidos a la detección y análisis de la posible amenaza y fases posteriores en las que predominan los factores motivacionales dirigidos a facilitar las acciones defensivas de lucha o huida (Blanchard y Blanchard, 1989; Fanselow, 1994; Lang, Davis y Öhman, 2000). Este modelo asume un circuito cerebral de activación de las reacciones defensivas cuyos principales centros serían el núcleo central de la amígdala y el núcleo del lecho de la estría terminal, los cuales, a través de sus conexiones con otras estructuras subcorticales, explicarían las diversas manifestaciones del miedo y la ansiedad (inmovilidad, hipervigilancia, reacciones vegetativas, potenciación del sobresalto motor, expresiones faciales de miedo, acciones defensivas). El modelo se inserta, a su vez, dentro de una concepción de las emociones (Bradley, 2000; Lang, 1995; Lang, Davis y Öhman, 2000) como disposiciones para la acción que se han ido desarrollando a lo largo de la evolución a partir de reacciones de carácter adaptativo ante situaciones relevantes para la supervivencia y que están relacionadas con los dos sistemas motivacionales primarios: el apetitivo, relacionado con las emociones positivas, y el defensivo, relacionado con las emociones negativas. La emoción se produciría cuando se activa cualquier estructura de información en el cerebro que conecte con estos sistemas motivacionales.

Este modelo se ha investigado en humanos utilizando como principal paradigma de estudio la visualización de imágenes afectivas superpuestas a la evocación del reflejo de sobresalto junto con el registro simultaneo de diversas respuestas psicofisiológicas tanto periféricas (frecuencia cardíaca, conductancia eléctrica de la piel, electromiografía de los músculos corrugador y cigomático) como centrales (EEG, potenciales eventorelacionados). Los resultados de numerosas investigaciones utilizando este paradigma muestran que la amplitud del reflejo de sobresalto, provocado por estimulación acústica repentina, se potencia cuando el organismo se encuentra procesando información emocionalmente desagradable o aversiva, mientras que la amplitud del sobresalto se inhibe cuando el organismo se encuentra procesando información emocionalmente agradable o apetitiva. Lang y colaboradores explican este fenómeno de acuerdo con su modelo de 'priming motivacional': los reflejos apetitivos y defensivos se potencian cuando existe congruencia entre el tipo de reflejo y el estado emocional en que se encuentra previamente el organismo (apetitivo o aversivo) mientras que los reflejos se inhiben cuando existe incongruencia entre el tipo de reflejo y el estado emocional del organismo (Lang, 1995).

\section{Resultados recientes sobre la defensa cardíaca}

La revisión de la literatura sobre la defensa cardíaca en humanos, utilizando como paradigma básico la presentación de un ruido intenso inesperado bajo diferentes condiciones de manipulación del estímulo y superposición de tareas (ver Vila et al., 2007), muestra los siguientes resultados:

1. La respuesta es un patrón complejo de cambios en la frecuencia cardíaca que, dependiendo del contexto y del individuo, muestra diferentes componentes acelerativos y desacelerativos a lo largo de los 80 segundos posteriores al inicio del estímulo. En grupos de personas no seleccionadas y sin tareas secundarias superpuestas, el patrón promedio se caracteriza por dos componentes acelerativos y dos componentes desacelerativos en orden secuencial alterno: aceleración-desaceleraciónaceleración-desaceleración.

2. Este patrón es provocado por estimulación acústica o electrocutánea intensa, pero no por estimulación visual intensa. La duración del estímulo es un factor crucial para su elicitación -no inferior a 500 milisegundos- mientras que el tiempo de subida del estímulo no lo es -tiempos de subida que eliminan el sobresalto motor pueden provocar el patrón completo de la respuesta.

3. La respuesta muestra una rápida habituación con la repetición del estímulo. La segunda aceleración/desaceleración es el componente que muestra la habituación más rápida. No obstante, la habituación se puede retrasar incrementando el intervalo temporal entre los estímulos, tanto dentro de la misma sesión como entre sesiones. De forma similar, la deshabituación puede facilitarse cambiando la cualidad sensorial del estímulo y su significación (por ejemplo, de ruido natural a ruido artificial).

4. Los mecanismos fisiológicos periféricos que controlan la respuesta incluyen influencias tanto simpáticas como parasimpáticas. La primera aceleración/desaceleración está controlada por influencias parasimpáticas: inhibición durante la primera aceleración y activación durante la siguiente desaceleración. La segunda aceleración/desaceleración está controlada por influencias simpáticas y parasimpáticas (estas últimas trabajando recíprocamente): activación simpática acompañada de inhibición parasimpática durante la segunda aceleración e inhibición simpática acompañada de activación parasimpática durante la segunda desaceleración. Los mecanismos fisiológicos corticales implicados en la respuesta apenas se han investigado. Los pocos datos existentes apuntan a una disminución de la potencia del ritmo alfa en la zona parieto- 
occipital coincidente con la primera aceleración/desaceleración (Guerra, 2007).

5. La respuesta está modulada tanto por factores atencionales como por factores emocionales: los componentes acelerativos de la respuesta se ven potenciados cuando los participantes realizan simultáneamente una tarea de atención externa (frente a una tarea de atención interna) y cuando los participantes se encuentran en un estado emocional negativo inducido por la visualización de imágenes desagradables o fóbicas. Por consiguiente, la defensa cardíaca, en contra de lo propuesto por el modelo cognitivo clásico, está más relacionada con incrementos en los mecanismos atencionales de procesamiento sensorial que con decrementos en dichos mecanismos.

6. La modulación emocional de la respuesta mediante la visualización de imágenes desagradables o fóbicas se manifiesta no sólo en la potenciación de los componentes acelerativos de la respuesta. Se observa, además, un cambio en la topografía de la respuesta: la primera desaceleración desaparece totalmente y la segunda aceleración se adelanta temporalmente observándose un único componente acelerativo seguido de una desaceleración final. Esta modulación se observa tanto bajo condiciones de presentación consciente de las imágenes como bajo condiciones de presentación no-consciente (enmascarada) de las imágenes.

7. Existen importantes diferencias individuales en el patrón de la respuesta pudiéndose identificar cuatro grupos de personas, dos predominantemente acelerativos y dos predominantemente desacelerativos. De los dos grupos con patrones predominantemente acelerativos, uno muestra el patrón típico con sus dos componentes acelerativo/desacelerativos secuenciales, el otro muestra un único componente acelerativo prolongado seguido de la desaceleración final. De los dos grupos con patrones predominantemente desacelerativos, uno muestra una desaceleración prolongada después de la primera aceleración, el otro muestra una vuelta a la línea de base después de la primera aceleración.

8. Estos patrones están asociados a diversos factores biológicos y psicológicos: ciclo menstrual, sexo, rasgos de personalidad (inestabilidad emocional, exceso de preocupación) y presencia de ansiedad patológica. Los patrones acelerativos se han encontrado en mujeres fóbicas durante la fase premenstrual (en comparación con la fase inter-menstrual), en hombres (en comparación con mujeres), en personas con altas puntuaciones en preocupación e inestabilidad emocional y en pacientes con trastornos de ansiedad. Un resultado especialmente relevante es la diferencia observada dentro de los dos patrones acelerativos: personas con altas puntuaciones en preocupación y pacientes con ansiedad generalizada tienden a mostrar el patrón acelerativo prolongado (sin la primera desaceleración) mientras que personas con alta ansiedad subclínica y pacientes con fobias específicas tienden a mostrar el patrón típico con sus dos componentes acelerativo/desacelerativos secuenciales.

\section{El modelo atencional-motivacional de la defensa cardíaca}

El patrón complejo de cambios en la frecuencia cardíaca característico de la reacción defensiva ante un estímulo aversivo inesperado puede entenderse dentro de la perspectiva naturalista de la cascada defensiva descrita anteriormente. Los dos componentes acelerativo/desacelerativos secuenciales parecen reflejar la sucesión de dos fases defensivas diferentes: una primera fase defensiva atencional, dirigida a interrumpir la actividad presente y analizar el peligro potencial, y una fase defensiva motivacional, dirigida a preparar al organismo para la defensa activa. De esta forma, el patrón de la defensa cardíaca, con sus dos componentes acelerativo/desacelerativos, representaría la transición de la atención a la acción: (a) primera aceleración/desaceleración: interrupción de la actividad presente y aumento de la atención hacia los estímulos externos; y (b) segunda aceleración/desaceleración: preparación para la defensa activa y recuperación si no ocurre un peligro real.

Esta secuencia, no obstante, puede alterarse si el estímulo provocador de la defensa cardíaca está precedido de señales de peligro (efecto de facilitación o priming) o si la persona se encuentra ya en un estado emocional negativo. Los datos encontrados sobre la modulación emocional de la defensa cardíaca y las diferencias individuales apoyan este punto de vista. El cambio topográfico en el patrón de la respuesta cuando el estímulo defensivo va precedido de la visualización de imágenes fóbicas o desagradables -transformado en una única aceleración aumentada y prolongada sin la desaceleración inicial- sugiere que la fase atencional ha sido ya activada por las señales de peligro precedentes y que la fase motivacional en preparación para las acciones defensivas se ha adelantado temporalmente. Esta misma interpretación puede aplicarse a las personas que muestran una única aceleración prolongada sin la desaceleración inicial en respuesta a estímulos aversivos inesperados. Este patrón de respuesta se ha encontrado en pacientes con ansiedad generalizada (Vila et al., 2007) y en personas con altas puntuaciones en preocupación excesiva (Delgado et al., 2009). Un estado pre-existente de alta ansiedad anticipatoria o alta preocupación puede actuar de la misma forma que la visualización de imágenes amenazantes justo antes de la presentación del estímulo defensivo, esto es, puede facilitar la reacción defensiva haciendo que se adelante la fase motivacional.

Este tipo de explicación de la defensa cardíaca permite no sólo integrar las dos aproximaciones tradicionales a la defensa cardíaca -la atencional y la motivacional-, sino también clarificar las descripciones contradictorias de la defensa cardíaca encontradas en la literatura. El modelo clásico (Graham, 1979, 1992; Graham y Clifton, 1966) describe la respuesta como una única aceleración mediada por la rama simpática del sistema nervioso vegetativo. Esta descripción parece ajustarse a los datos sobre la defensa cardíaca cuando el estímulo aversivo está facilitado por señales de peligro anticipatorias o por un estado pre-existente de ansiedad o preocupación. Bajo estas 
condiciones el patrón de la respuesta se convierte en una única aceleración aumentada y prolongada. Por tanto, dependiendo de que el estímulo defensivo esté facilitado o no lo esté, la defensa cardíaca puede describirse como una única aceleración mediada simpáticamente o como un patrón complejo de cambios acelerativos y desacelerativos mediados por influencias tanto simpáticas como parasimpáticas

El modelo, además, permite explicar los dos patrones desacelerativos encontrados en algunos individuos. La prolongada desaceleración después de la aceleración inicial puede deberse a una activación parasimpática sostenida durante la evocación de la respuesta. Bradicardias defensivas se han encontrado asociadas a inmovilidad (Azevedo et al., 2005; Fachinetti et al., 2006) y a la percepción de imágenes desagradables (Bradley, 2000). El llamado estilo defensivo reactivo descrito en la literatura sobre estrés (Frankenhaeuser, 1986; Koolhass et al., 1999) también se caracteriza por ir acompañado de inmovilidad y baja activación simpática. Por consiguiente, puede hipotetizarse que las personas que responden a estimulación intensa con una bradicardia prolongada después de la aceleración inicial responden defensivamente con un patrón de respuesta típico del estilo defensivo pasivo (predominio de la fase atencional), mientras que aquellos que responden con el patrón acelerativo prolongado serían personas que responden defensivamente con un patrón de respuesta típico del estilo defensivo activo (predominio de la fase motivacional). Las personas que responden con un retorno a la línea de base inmediatamente después de la primera aceleración serían personas con una reacción defensiva reducida.

\section{Conclusión}

El estudio de las reacciones defensivas constituye una de las principales vías para avanzar el conocimiento sobre los mecanismos del miedo y la ansiedad. Aunque la investigación en este campo ha sido abundante a lo largo de los últimos años, todavía se desconocen muchos aspectos básicos relativos a las reacciones defensivas en humanos. En el ámbito de la defensa cardíaca, por ejemplo, se desconocen los mecanismos corticales y vegetativos propios de los difentes patrones acelerativos y desacelerativos descritos en los apartados anteriores así como su papel en los estilos defensivos activo/pasivo o en los trastornos de la ansiedad. La investigación futura deberá avanzar en esta dirección.

\section{Referencias}

Azevedo, T. M., Volchan, E., Imbiriba, L. A., Rodrigues, E. C., Oliveira, J. M., Oliveira, L. F., Lutterbach, L. G. y Vargas, C. D. (2005). A freezing-like posture to pictures of mutilation. Psychophysiology, 42, 255-260.

Blanchard, R. J. y Blanchard, D. C. (1989) Attack and defense in rodents as ethoexperimental models for the study of emotion. Progress in Neuro-Psychopharmacology and Biological Psychiatry, 13, 3-14.

Bond, D. D. (1943). Sympathetic and vagal interaction in emotional responses of the heart rate. American Journal of Physiology, 138, 468-478.

Bradley, M. M. (2000). Emotion and motivation. En J. T. Cacioppo, L. G. Tassinary y G. G. Berntson (Eds.) (2000). Handbook of psychophysiology. New York: Cambridge University Press.

Cannon, W. B. (1929). Bodily changes in pain, hunger, fear, and rage. New York: Reinhold.

Cloete, N. (1979). Autonomic responsivity of subjects with body boundary differences during white noise stimulation. Acta Psicologica, 43, 177-183.

Davis, M. (1992): The role of amygdala in fear potentiated startle. Implications for animal models of anxiety. Trends in Pharmacological Science, 13: 35-41.

Dawson, M. E., Schell, A. M. y Böhmelt, A. H. (1999). Startle modification: Implications for neuroscience, cognitive science and clinical science. New York: Cambridge University Press.

Delgado, L. C., Guerra, P., Perakakis, P., Mata, J. L., Pérez, M. N. y Vila, J. (2009). Psychophysiological correlates of chronic worry: cued versus non-cued fear reaction. International Journal of Psychophysiology, 74, 280-287.

Dienstbier, R. A. (1989). Arousal and physiological toughness: implications for mental and physical health. Psychological Review, 96, 84-100.

Eves, F. F. y Gruzelier, J. M. (1984). Individual differences in the cardiac response to high intensity auditory stimulation. Psychophysiology, 21, 342-352.

Eves, F. F. y Gruzelier, J. M. (1987). Individual differences in the vascular components of the defensive response in humans. Journal of Psychophysiology, 1, 161-172.

Fanselow, M. S. (1994). Neural organization of the defense behaviour system responsible for fear. Psychonomic Bulletin and Review, 1, 429-438.

Facchinetti, D. D., Imbiriba, L. A., Azevedo, T. M., Vargas, D. D. y Volchan, E. (2006). Postural modulation induced by pictures depicting prosocial or dangerous contexts. Neuroscience Letters, 410, 52-56.

Frankenhaeuser, M. (1986). A psychobiological framework of research on human stress and coping. En M.Appleyy R. Trumbull (Eds.), Dynamics of stress. New York: Plenum Press.

Graham, F. K. (1979). Distinguishing among orienting, defense and startle reflexes. En H. D. Kimmel, E. H. Van Olst y J. F. Orlebeke (Eds.), The orienting reflex in humans. New Jersey: Erlbaum.

Graham, F. K. (1992). The heartbeat, the blink, and the brain. En B. A. Campbell, H. Hayne y R. Richardson (Eds.), Attention and information processing in infants: Perspectives from human and animal research (pp. 3-29). Hillsdale, NJ: Erlbaum. 
Graham, F. K. y Clifton, R. K. (1966). Heart-rate change as a component of the orienting response. Psychological Bulletin, 65, 305-320.

Guerra, P. (2007). Componentes periféricos y centrales de la atención y las respuestas defensivas. Tesis Doctoral. Universidad de Granada.

Jung-Stalmann, B. (2003). The cardiac defense response: personality and stress management. Hamburg: Logos Verlag Berlin.

Knott, V. J. y Bulmer, D. R. (1984). Heart rate responsivity to a high intensity auditory stimulus: a comparison of male alcoholics and normal controls. Addictive Behaviors, 9, 201-205.

Koolhaas, J. M., Korte, S. M., de Boer, S. F., van der Vegt, B. J., van Reenen, C. G., Hopster, H., de Jong, I. C., Ruis, M. A. W. y Blokhuis, H. J. (1999). Coping styles in animals: current status in behavior and stress-physiology. Neuroscience and Behavioral Reviews, 23, 925-935.

Lacey, J. I. (1972). Some cardiovascular correlates of sensorimotor behavior: example of visceral afferent feedback? En C. H. Hockman (Ed.), Limbic system mechanisms and autonomic function. Springfield, Il.: Thomas.

Lang, P. J. (1995). The emotion probe: Studies of motivation and attention. American Psychologist, 50, 372-385.

Lang, P. J., Bradley, M. M. y Cuthbert, B. N. (1997). Motivated attention. En P. J. Lang, R. F. Simons y M. Balaban (Eds.) (1997), Attention and orienting: sensory and motivational processes. Hillsdale, N.J.: Erlbaum.

Lang, P. J., Davis, M. y Öhman, A. (2000). Fear and anxiety: Animal models and human cognitive psychophysiology. Journal of Affective Disorders, 61, 137-159.

LeDoux, J. E. (2000). Emotion circuits in the brain. Annual Review of Neuroscience, 23, 155-184.

Lovallo, W. R. y Gerin, W. (2003). Psychophysiological reactivity: mechanisms and pathways to cardiovascular disease. Psychosomatic Medicine, 65, 36-45.

Obrist, P. A. (1981). Cardiovascular psychophysiology: A perspective. New York: Plenum Press.

Öhman, A., Hamm, A. y Hugdahl, K. (2000). Cognition and the autonomic nervous system: Orienting, anticipation, and conditioning. En J. Cacioppo, L. Tassinary y G. G. Berntson (Eds.), Handbook of psychophysiology. New York: Cambridge University Press.
Pavlov, I. (1927). Conditioned reflexes. Oxford: Oxford Univeristy Press.

Richards, M. y Eves, F. (1991). Personality, temperament and the cardiac defense response. Personality and Individual Differences, 7, 999-1007.

Ruiz-Padial, E. y Vila, J. (2007). Fearful pictures not consciously seen modulate the startle reflex in human beings. Biological Psychiatry, 61, 996-1001.

Schauer, M., Neuner, F. y Elbert, T. (2005). Narrative Exposure Therapy. A short-term intervention for traumatic stress disorder after war, terror or torture. Göttingen, Germany: Hogrefe \& Huber

Selye, H. (1956). The stress of life. New York: McGraw-Hill.

Sokolov, E. N. (1963). Perception and the conditioned reflex. Elmsford, NY: Pergamon Press.

Steptoe, A. y Vögele, C. (1991). Methodology of mental stress testing in cardiovascular research. Circulation, 83, 14-24.

Turner, J. R. (1994). Cardiovascular reactivity and stress: Patterns of physiological response. New York: Plenum Press.

Turpin, G. (1986). Effects of stimulus intensity on autonomic responding: The problem of differentiating orienting and defense reflexes. Psychophysiology, 23, 1, 1-14.

Turpin, G. y Siddle, D. A. (1978). Cardiac and forearm plethysmographic responses to high intensity auditory stimulation. Biological Psychology, 6, 267-281.

Turpin, G. y Siddle, D. A. (1983). Effects of stimulus intensity on cardiovascular activity. Psychophysiology, 20, 611-624.

Vila, J., Fernández, M. C., Pegalajar, J., Vera, M. N., Robles, H., Pérez, N., Sánchez, M. B., Ramírez, I., y Ruiz-Padial, E. (2003). A new look at cardiac defense: Attention or emotion? The Spanish Journal of Psychology, 6, 60-78.

Vila, J., Guerra, P., Muñoz, M. A., Vico, C., Viedma-del Jesús, M. I., Delgado, L. C., Perakakis, P., Kley, E., Mata, J. L. y Rodríguez, S. (2007). Cardiac defense: From attention to action. International Journal of Psychophysiology, 66, 169-182.

Fecha de recepción: 9 de octubre de 2009 Fecha de aceptación: 24 de noviembre de 2009 